\title{
Fuzzy Control of Batteries Fast Charging for HEV in Regenerative Braking
}

\author{
Zhumu FU ${ }^{1, a^{*}}$ and Aiyun $\mathrm{GAO}^{2, \mathrm{~b}}$ \\ ${ }^{1}$ College of Information Engineering, Henan University of Science and Technology, Luoyang 471023, \\ P. R. China; \\ ${ }^{2}$ College of Vehicle and Traffic Engineering, Henan University of Science and Technology, Luoyang \\ 471023, P. R. China \\ afzm1974@163.com, bgao_cloud@163.com
}

Keywords: HEV; regenerative braking; fuzzy control; fast charging.

\begin{abstract}
In this paper, a fuzzy controller is constructed on the basis of the hybrid electric vehicle's (HEV's) batteries fast charging during regenerative braking. The proposed method is based on Mascc law and the mathematical relationship between open circuit voltage with the internal resistance model and charge efficiency. A closed-loop control system simulation model is accomplished in Matlab and the rate of electric energy recovery for HEV is calculated with different control strategy. It is shown that the rate of electric energy recovery with the fuzzy control strategy is increased by $8.41 \%$ compared with the constant current charge method in the same brake condition, thus the fuel economy is greatly improved.
\end{abstract}

\section{Introduction}

Batteries charging management is an effective method that can improve the efficiency of braking energy recovery, and reduce fuel consumption and ensure batteries' safety during the regenerative braking of hybrid electric vehicles (HEVs) [1]. Regenerative braking has become an important subject for HEVs [2, 3]. In recent years, some breakthroughs [4-5] have been made worldwide in the field of batteries management system and fast charging. Malekjamshidi et al [6] studied the battery charged intelligent system, taking into account the temperature control and fast charging requirements of batteries. $\mathrm{Xu}$ et al [7] applied the Kalman filtering theory to the estimation of the SOC by using a stochastic fuzzy neural network to model the battery nonlinear dynamic. Qiang et al [8] proposed a new combined SOC estimation method, in which coulomb-accumulation and resistance-capacitor model were weighted to estimate the SOC for HEVs. However, the drawback of these methods is that there are many variables' online data to test, which may lead to some inaccuracies.

Most studies in the batteries fast charging have focused on the research of battery formula, and few studies have reported research results especially suitable for urban road braking of HEVs or the fast charging in combination with regenerative braking. Therefore, according to the Mascc law and combined with the characteristics of the HEV during regenerative braking [9, 10], this paper designs a fuzzy control strategy of batteries fast charging during regenerative braking, which not only ensures the safety of fast charging by taking fully into account the temperature rise of batteries but also is suitable for batteries fast charging of HEVs in city condition. Thus, the rate of electric energy recovery is increased obviously and the maximum regenerative energy is obtained.

The remainder of this paper is organized as follows. Section 2 designs the models of batteries fast charging for HEVs during regenerative braking. Control strategy and the design of fuzzy logic controller (FLC) are presented in Sections 3. Validation of the control strategy proposed and simulation results are discussed in Section 4. Section 5 highlights this paper's key conclusions. 


\section{Modeling of Batteries Fast Charging for HEVs during Regenerative Braking}

Batteries' Heat Transfer Theory Models. The temperature rise of batteries fast charging is a main influence factor on charging safety. Therefore, a model of batteries' heat-transfer theory is built to estimate the temperature rise within the scope of the security. By neglecting the side effect of batteries fast charging, then the heat transfer model of batteries is determined by resistance influence, thermal conductivity and air convective heat transfer and other factors.

The surface temperature of batteries case $T_{\text {bat }}$ can be calculated according to equations Eq. 1- 3, which can be found in Eq. 4.

According to the law of conservation of energy, the heat from batteries charging is equal to the energy including energy loss of resistance and energy coming from chemical reaction of batteries charging. The heat from batteries charging $Q_{\mathrm{w}}$ is expressed as follows.

$$
Q_{\mathrm{w}}=\int_{0}^{t}\left(0.152 I_{\mathrm{b}}+I_{\mathrm{b}}^{2} R_{\mathrm{b}}\right) d t
$$

Where, $I_{\mathrm{b}}$ is the actual charging current, and $R_{\mathrm{b}}$ is the resistance of batteries charging.

$$
Q_{\text {out }}=\frac{T_{\text {bat }}-T_{\text {air }}}{R_{\mathrm{th}}}
$$

Where, $Q_{\text {out }}$ is the heat dissipation of batteries, $T_{\text {air }}$ is ambient temperature, $R_{\mathrm{th}}$ is the effective thermal resistance of batteries and is expressed as below.

$$
R_{\mathrm{th}}=\frac{1}{h A}+\frac{1}{\lambda A}
$$

Where, $A$ is the surface size of batteries case, $\lambda$ is the thermal conductivity of batteries case material, and $h$ is the air convection coefficient.

In conclusion, batteries' heat transfer theory model is made as follows.

$$
T_{\text {bat }}=T_{0}+\int_{0}^{t} \frac{Q_{\mathrm{w}}-Q_{\text {out }}}{m_{\mathrm{b}} C_{\mathrm{b}}} d t
$$

Where, $T_{0}$ is the initial surface temperature of batteries case, $m_{\mathrm{b}}$ is the batteries mass, and $C_{\mathrm{b}}$ is the specific heat coefficient of the batteries.

The open circuit voltage with the internal resistance models of batteries. The SOC is a key parameter in the process of batteries fast charging. The SOC will vary with both thermodynamic effect and quantum effect, so it is not easy to estimate SOC with accurate mathematical models. Most models are the empirical or semi-empirical models based on some experimental data. Considering the temperature influence on batteries' voltage and resistance, the SOC of batteries is estimated by using an open circuit voltage with the internal resistance model. The circuit can be shown in Figure 1.

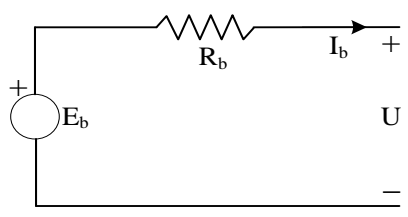

Figure 1 The Equivalent Circuit Model of Batteries

As shown in Figure 1, by neglecting the inductance of circuit and assuming that the charging current has no undulating current, the charging voltage $U$ is expressed as

$$
U=E_{\mathrm{b}}-I_{\mathrm{b}} R_{\mathrm{b}}
$$

Where, $E_{\mathrm{b}}$ represents open-circuit voltage, $I_{\mathrm{b}}$ is negative in the charging process, $R_{\mathrm{b}}$ is charging resistance and $R_{\mathrm{b}}=f\left(T_{\text {bat }}, S O C\right)$.

According to the relationship among the power $P_{\mathrm{b}}$, voltage and electric current, an equation is expressed as 


$$
P_{\mathrm{b}}=U I_{\mathrm{b}}
$$

By combining Eq. 5 with Eq. 6, considering $I_{\mathrm{b}}$ is negative, the efficiency of charging $\eta_{\mathrm{b}}$ is calculated as

$$
\eta_{\mathrm{b}}=\frac{E_{\mathrm{b}} I_{\mathrm{b}}}{P_{\mathrm{b}}}=\frac{E_{\mathrm{b}} I_{\mathrm{b}}}{\left(E_{\mathrm{b}}-I_{\mathrm{b}} R_{\mathrm{b}}\right) I_{\mathrm{b}}}
$$

In the process of charging, SOC is closely related to charging current ${ }^{\mathrm{b}}$. On the basis of SOC definition of batteries [8], there is the following equation.

$$
S O C=S O C_{0}+\frac{k_{T_{\mathrm{b}}} \int_{0}^{t} I_{\mathrm{b}} \eta_{\mathrm{b}} d t}{C_{\mathrm{t}}}
$$

Where, $k_{T_{\mathrm{b}}}$ is temperature coefficient, while ambient temperature is $25^{\circ} \mathrm{C}, k_{T_{\mathrm{b}}}=1$; $C_{\mathrm{t}}$ is the capacity of batteries.

The batteries' heat transfer theory models and the open circuit voltage with the internal resistance models reflect the relationship among the charging voltage, charging current and the efficiency of charging, and SOC is also closely related to ${ }^{\mathrm{b}}$. As for these factors, SOC and the surface temperature of batteries case $T_{\text {bat }}$ are considered as the inputs to the FLC while the output of the FLC is the maximum charging current $I_{\mathrm{fe}}$.

\section{Control Strategy for Batteries Fast Charging}

The calculation of actual charging current. Under the city driving cycle, the time of regenerative braking for HEVs is less than 30s. So it needs batteries fast charging. In this paper, maximum limited current is established ensuring the batteries safety. According to Mascc law, the equations are represented as follows.

$$
\begin{gathered}
I=I_{0} e^{-a t} \\
a=I_{0} /\left(C_{\mathrm{t}}-C_{\mathrm{r}}\right)
\end{gathered}
$$

Where, $I$ is the maximum current at time $t ; I_{0}$ is the designed current when $t=0$, which is decided by the character of batteries; $a$ is the coefficient of charging; $C_{\mathrm{r}}$ is the initial capacity of batteries.

This paper uses NIMH batteries system, which includes 120 modules with nominal cell voltage of $1.2 \mathrm{~V}$. When $\mathrm{SOC}=0.4$, the ideal charging current $I_{0}=80$ A. According to Mascc law, we assume that the NIMH batteries system is charged from $\mathrm{SOC}=0.4$ to $\mathrm{SOC}=0.8$ in 10 minutes, so $a \approx 0.0085$. The associated charging curves are shown in Figure 2.

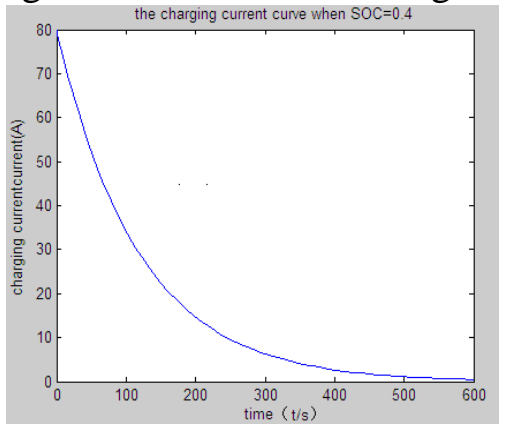

(a) The Charging Current Curve

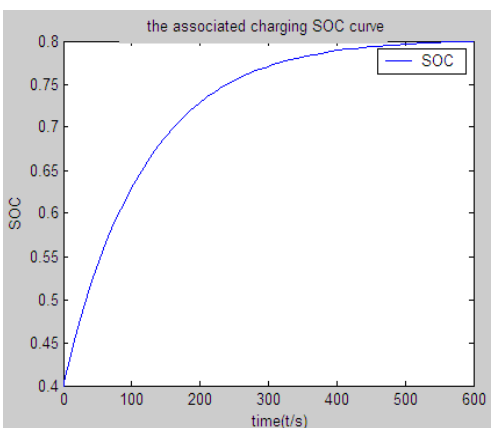

(b) The Associated Charging SOC Curve

Figure 2 The Charging Current Curve and SOC Curve according to Mascc Law.

Figure 2(a) shows the charging current curve with the initial SOC $=0.4$. It is not allowed that the charging current is above the curve. Or else, the batteries would be damaged over time because their 
temperature rises quickly. For this reason, the value set of the charging current must be under the curve. According to Figure 2(b), we can get any ideal value of charging current in accord with different SOC. However, the working space of batteries in HEVs is narrow and there are many nonlinear factors in reality. To ensure the safety of batteries, the ideal current will be given a division operation. The actual current is determined by following equation.

$$
I_{\mathrm{ac}}=I_{0} / 1.3
$$

Where, $I_{\mathrm{ac}}$ is the maximum current assumed in reality, so the initial current is $62 \mathrm{~A}$ in accord with initial $S O C=0.4$.

When braking in city condition, the charging system of HEVs is influenced by driver's demand, driving cycle and other nonlinear factors. Since these factors also lead to the imprecise model, so a fuzzy logic control algorithm will be suit for the model. According to the related parameters, a fuzzy controller can be used to estimate the max charging current $I_{\mathrm{fe}}$.

In the process of batteries charging, the actual charging current is related to the braking power. A theoretical charging current $I_{\text {th }}$ can be determined by the braking power.

$$
\begin{gathered}
P_{\mathrm{ch}}=\eta_{\mathrm{d}}\left[\frac{1}{2} m v^{2}(t-1)-\frac{1}{2} m v^{2}(t)\right] \\
I_{\mathrm{th}}=\eta_{\mathrm{ch}} P_{\mathrm{ch}} / U
\end{gathered}
$$

where $P_{\mathrm{ch}}$ is the power of regenerative braking, $\eta_{\mathrm{d}}$ is the ratio of the regenerative braking power to sum of the braking power, $\eta_{\mathrm{d}}<1, m$ is the mass of the HEV, $v(t)$ and $v(t-1)$ is the vehicle velocity at instant time $t$ and $(t-1), \eta_{\mathrm{ch}}$ is the efficiency of charging.

By using $I_{\text {th }}$, the maximum charging current $I_{\text {fe }}$, which is estimated by the designed fuzzy controller. We can calculate the actual charging current $I_{\mathrm{b}}$, the equation is represented as follows.

$$
I_{\mathrm{b}}=\min \left(I_{\mathrm{fe}}, I_{\mathrm{th}}\right)
$$

The fuzzy logic controller Design. The fuzzy logic controller adopts two-dimensional input structure, corresponding to the battery charge state SOC and the temperature $T_{\text {bat }}$. The output of the controller is the estimated current $I_{\mathrm{b}}$. The exact amount of domain of the SOC is [0 0.8]; the temperature $T_{\text {bat }}$ is [0 62]; and the estimated current $I_{\mathrm{b}}$ is [0 45]. To make the fuzzy controller suit for reality, we make the universe of discourse in the range [0 1] for inputs and output, and design the quantization factors $K_{s o c}=1.25, K_{\text {tem }}=1 / 45, K_{I}=1 / 62$.

The domains of fuzzy inputs SOC, $T_{\text {bat }}$ and output $I_{\mathrm{b}}$ are $\operatorname{SOC}\{S S, S M, S B, M, B S, B M, B B\}$, $T_{\text {bat }}\{S, S M, M, B M, B\}$, and $I_{\mathrm{b}}\{V S S, S S, B S, S M, S B, V B B, B B, B M, M\}$. Membership functions use two opening trapezoid membership functions in the both sides and triangle membership functions in the middle.

This paper uses the popular Mamdani algorithm and the largest average method (MOM) fuzzy reasoning by experiences and simulation. The minimum SOC value of the battery should be controlled above 0.2 , and charging should be prohibited while the SOC value is too high. We can make conclusions by the relationship between the input and output: the lower the temperature is and the smaller the SOC value of the battery is, the larger the acceptable charging current is. On the contrary, the higher the temperature is and the larger SOC value is, the smaller the acceptable charging current is, until it is zero.

After ascertaining each input and output linguistic variables and membership functions, the rules of the fuzzy controller are designed and further slightly modified based on the simulation results. The rules of fuzzy control are shown in Table. 1. 
Table 1 The Rules of Fuzzy Control

\begin{tabular}{|l|l|l|l|l|l|l|l|}
\hline$I_{\mathrm{b}}$ & SOC & SM & SB & M & BS & BM & BB \\
\hline$T_{\text {bat }}$ & & & & & & & \\
\hline S & VBB & VBB & BB & BM & BS & M & SB \\
\hline SM & BB & BB & BM & BS & M & SB & SM \\
\hline M & BM & BM & BS & M & SB & SM & SS \\
\hline BM & BM & BS & M & SB & SM & SS & SS \\
\hline B & BS & BS & M & SB & SM & SS & VSS \\
\hline
\end{tabular}

\section{Simulation and Comparative Analysis}

This paper makes the simulation to compare the constant current charge method with the designed fast charging fuzzy control strategy. During regenerative braking, the rate of electric energy recovery $\varepsilon$ is calculated by the following equation.

$$
\varepsilon=\frac{\int_{0}^{t}(U-I(t) R(t)) I(t) d t}{m v_{0}^{2} / 2}=\frac{\Delta E_{\mathrm{b}}}{m v_{0}^{2} / 2}
$$

Where, $\Delta E_{\mathrm{b}}$ is the energy of HEV during regenerative braking, $I(t)$ and $R(t)$ are the charging current and the internal resistance of the battery respectively at time $t$, and $v_{0}$ is the initial vehicle braking velocity.

The simulation comparisons of the designed fuzzy control strategy with the constant current charge method are illustrated in Figure 3.

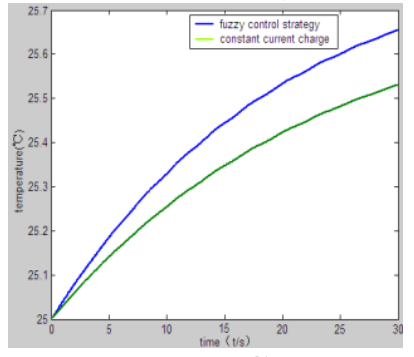

(a) Temperature Curve

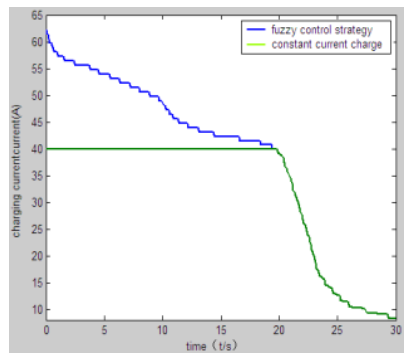

(b) Charging Current Curve (c) SOC Values Variation Vurve

Figure 3 Comparison of Simulation Results

At the early stage, the charging current of batteries is relatively larger by using fuzzy control strategy, so their temperature is rising quickly than by adopting the constant current charge method, but they are in the range of safety according to reference [8] as shown in Figure 3 (a) and (b). At the later stage, Eq. 12- 14 show that the charging current of batteries becomes small with the vehicle velocity decreasing, and the charging current equals by using the two control strategies. Figure 3(c) shows the change of SOC value. The rate of pre-charging SOC value change is significantly greater by using fuzzy control strategy than constant current charge method and at the later stage (after 19s), consistency with the slope of the two curves indicates that the rate of SOC change is consistent, which proves the more energy by regenerative braking system. Therefore, the validity and rationality of the designed fuzzy control strategy are proved.

According to Eq. 15, it can calculate energy recovery rate of fuzzy control strategy and constant current control charging, respectively. The sum energy of recovery is $219024 \mathrm{~J}$ and the energy recovery rate is $42.05 \%$ with fuzzy control rapid strategy, while the sum energy of recovery is $175219 \mathrm{~J}$ and the energy recovery rate is $33.64 \%$ by adopting the constant current. It shows that the energy recovery of the fuzzy control strategy improves $8.41 \%$ than that of constant current charge method and it proves the high efficiency of the designed fuzzy control strategy. 


\section{Conclusion}

This paper puts forward a fuzzy control strategy of batteries fast charging for HEVs during regenerative braking to improve the fuel economy. The design of the corresponding fuzzy controller is described in detail based on the mathematical and simulation models. The results show that the HEV with the designed control strategy can obtain the maximum regenerative energy, so it has good fuel economy on the basis of charging safety. However, the driving cycle is very complex and diverse. Therefore, in the practical application, combining other intelligent technologies with fuzzy control strategy designed for batteries fast charging and overcoming the dynamics disturbance will become the focus of future study.

\section{Acknowledgements}

The authors would like to thank the anonymous reviewers for their constructive and insightful comments for further improving the quality of this note. This work was partially supported by National Natural Science Foundation of China under Grant Nos. 61473115, 51277116 and 51375145, China Postdoctoral Science Foundation under Grant No. 2013T60670, Science and Technology Innovative Foundation for Distinguished Young Scholar of Henan Province under Grant No. 144100510004, the Science and Technology Programme Foundation for the Innovative Talents of Henan Province University Grant No. 13HASTIT038 and the Key Scientific and Technological Project of Henan Province Grant No. 132102210247.

\section{References}

[1] Yan J Y, Xu G Q, Qian H H, et al. Model predictive control-based fast charging for vehicular batteries [J]. Energies, 2011, 4(8): 1178-1196.

[2] Jo C, Yeo T, Hwang S, et al. Cooperative regenerative braking control algorithm for an automatic-transaction- based hybrid electric vehicle during a downshift [J]. Journal of Automobile Engineering, 2012, 226(4): 457-467.

[3] Qin D T, Ye M, Liu Z J. Regenerative braking control strategy in mild hybrid electric vehicles equipped with automatic manual transmission [J]. Frontiers of Mechanical Engineering In China, 2008, 2(3): 364-369.

[4] Wai R J, Jhung S J, Liaw J J, et al. Intelligent Optimal Energy Management System for Hybrid Power Sources Including Fuel Cell and Battery[J]. IEEE Transactions on Power Electronics, 2013, 28(7): 3231-3244.

[5] Johnson J, Chowdhury M, He Y M, et al. Utilizing real-time information transferring potentials to vehicles to improve the fast-charging process in electric vehicles [J]. Transportation research Part C: Emerging technologies, 2013, 26C (Jan.): 352-366.

[6] Malekjamshidi Z, Jafari M. Design, Simulation and Implementation of an Intelligent Battery Charging System [C]. 2009 International Conference on Computer and Electrical Engineering, ICCEE 2009, 2009, 2(2009): 242-246.

[7] Xu L, Wang J P, Chen Q S. Kalman filtering for state of charge estimation for battery management system based on a stochastic fuzzy neural network battery model [J]. Energy Conversion and management, 2012, 53(1): 33-39.

[8] Qiang J X, Ao G Q, Yang L. Estimation Method on the Battery State of Charge for Hybrid Electric Vehicle [J]. Chinese Journal of Mechanical Engineering, 2008, 21(3): 20-25.

[9] Duval-Destin M, Kropf T, Abadie V, et al. Impacts of an electric powertrain on the breaking system [J]. AutoTechnology, 2011, 11(4): 28-33.

[10] Wang Z, Ma C Y, Wang F. An Analysis on the Braking Energy Regeneration in Electromechanical Braking System [J]. Qi che Gong cheng, 2010, 32(11):972-976. 